\title{
Generating Fiber Crossing Phantoms Out of Experimental DWIs
}

\author{
Matthan Caan ${ }^{1,2}$, Anne Willem de Vries ${ }^{2}$, Ganesh Khedoe ${ }^{2}$, Erik Akkerman ${ }^{1}$, \\ Lucas van Vliet ${ }^{2}$, Kees Grimbergen ${ }^{1}$, and Frans Vos $^{1,2}$ \\ ${ }^{1}$ Department of Radiology, Academic Medical Center, \\ University of Amsterdam, NL \\ ${ }^{2}$ Quantitative Imaging Group, Delft University of Technology, NL \\ m.w.a.caan@tudelft.nl
}

\begin{abstract}
In Diffusion Tensor Imaging (DTI), differently oriented fiber bundles inside one voxel are incorrectly modeled by a single tensor. High Angular Resolution Diffusion Imaging (HARDI) aims at using more complex models, such as a two-tensor model, for estimating two fiber bundles.

We propose a new method for creating experimental phantom data of fiber crossings, by mixing the DWI-signals from high FA-regions with different orientation. The properties of these experimental phantoms approach the conditions of real data. These phantoms can thus serve as a 'ground truth' in validating crossing reconstruction algorithms. The angular resolution of a dual tensor model is determined using series of crossings, generated under different angles. An angular resolution of $0.6 \pi$ was found in data scanned with a diffusion weighting parameter $b=1000$ $\mathrm{s} / \mathrm{mm}^{2}$. This resolution did not change significantly in experiments with $b=3000$ and $5000 \mathrm{~s} / \mathrm{mm}^{2}$, keeping the scanning time constant.
\end{abstract}

\section{Introduction}

In the last decade, specifications of MR-scanners have increasingly improved. Consequently, Diffusion Weighted Images (DWIs) can be acquired at higher resolution and with better image quality. A more precise analysis of the diffusion profile of water in the human brain has thus become possible. This diffusion process is modeled by a rank-two symmetric positive-definite tensor [1, describing both the average distance and the orientation of the displacement. Anisotropic diffusion is associated with white matter structures, whereas isotropic diffusion is generally related to grey matter and cerebral spinal fluid. The Fractional Anisotropy (FA) has been introduced as a distance measure to an isotropic tensor [2], which is of use in analyzing changes in white matter integrity. The first eigenvector of the diffusion tensor represents the orientation of fibrous white matter structures, which can be tracked throughout the image volume 3. Such tractography is of practical interest for pre-operative planning. Additionally, studies to brain diseases are increasingly using fiber tracts as features in analysis 4 .

Still, the minimal size of a voxel in a DWI is orders of magnitude higher than the diameter of the individual axons. Only macroscopic brain properties 
can therefore be described. What is more, it is likely that multiple fiber bundles are crossing inside a single voxel, due to the partial volume effect. Multiple anisotropic diffusion processes in different directions are then modeled as a single process, introducing a severe bias in the tensor estimation. Consequently, regions of crossing fibers have to be excluded from comparative studies to brain diseases using tensor properties. Additionally, the measured principal diffusion direction is ill-defined and not related to the underlying anatomical structure. Fiber tracking based on a single tensor fit will erroneously connect distinct tracts or be terminated in a crossing. A dual tensor fit in regions of crossing fibers is thus indispensable for correctly tracking fibers through the brain.

Several solutions were proposed for overcoming the crossing-fiber problem. Nonparametric methods aim to find the probability density function of the spin diffusion displacement. For instance, $q$-ball imaging was proposed [5] for calculating diffusion orientations from High Angular Resolution Diffusion Images (HARDI). Parametric methods include a two-tensor model for analyzing the diffusion signal [5 6 7]. This work was continued by using spherical harmonics to characterize anisotropy [8], which was elsewhere shown to be related to the coefficients of higher order tensors [9]. Recently, high angle fiber crossings were reconstructed in clinical data with a diffusion weighting parameter $b=1000 \mathrm{~s} / \mathrm{mm}^{2}$ [10]

In order to validate the reconstruction of crossings, various experiments with simulated data have been performed. For instance, data with additive uncorrelated Gaussian noise was simulated 11. Scanning artifacts and patient movement are thereby ignored. Additionally, using a spinal chord, an experimental crossing phantom was created [12]. Still, a ground truth is missing for properly facilitating a validation of crossings found in the human brain.

We propose a new method for generating fiber crossing phantoms out of experimental data, containing realistic imaging artifacts. These phantoms can serve as a 'ground truth' in validating algorithms aiming to resolve crossing fibers. Series of crossings, generated under different angles, give insight into the precision that can be achieved in estimating the fiber bundle orientations. Therefore, a restricted two-tensor model is fitted on the projected data onto the plane of the crossing. Included in our analysis is the influence of the diffusion weighting parameter $b$ on the angular resolution.

Finally, the relevance of a proper modeling of crossing fiber regions is demonstrated. Streamlines generated through a crossing are shown to erroneously depict the fiber paths. Using a dual tensor model, it is shown that the FA of two crossing fibers bundles can be distinctively estimated.

\section{Method}

\subsection{Experimental Phantom Data}

Signal. The MR-signal measured in a certain voxel contains the contributions of the individual underlying volume fractions of different tissue. Dependent on the heterogeneity of the local tissue, more or less distinct fractions are present 
within a voxel. A summation of the fractions is assumed to correctly model the acquired MR-signal. This assumption is justified by the fact that there is no reason to expect inherent phase signal differences within a voxel. Previously, this summability was for instance silently used in [13, where an MR-signal is Monte Carlo simulated by aggregating a number of spins per voxel. And as an illustration, the figures 10 and 2 $\mathrm{b}$ can be compared, showing that adjacent perpendicular fiber bundles contribute equally to the signal of the intermediate voxel, inducing partial voluming.

We now propose to mix voxels out of experimental Diffusion Weighted Images (DWIs) whose FA is high enough to assume a single fiber bundle inside. Thus we are able to generate fiber crossings with different known properties, derived from single tensor fits of the individual parts. Here, only two voxels are mixed; dependent on the application even a higher number of orientations could be combined.

By using this approach, most known and unknown imaging artifacts that are present in real data are included in the phantoms. A realistic validation of a crossing reconstruction algorithm of interest has thus become possible.

Noise. Diffusion is measured as an attenuated signal, such that noise is expected to significantly affect the acquired images. This noise follows a Gaussian distribution, added independently to both the real and imaginary part of the signal. DWIs, being magnitude images with relatively low SNR, are thereby containing Rician noise [14. The noise in the generated phantom data now yields the sum of two Rician noise processes. Following the central limit theorem, this noise distribution can be expected to slightly tend back towards Gaussian. For simplicity, a Gaussian noise model is now used in reconstructing the generated crossing fibers.

\subsection{Two-Tensor Model}

In the case of two fiber orientations in a single voxel, distinction between these orientations is needed. We model the diffusion process of two fibers as the sum of two Gaussian distributions with different diffusion tensors, $\mathbf{D}^{1}$ and $\mathbf{D}^{2}$, closely following [15. Note that this model intuitively closely relates to the way the experimental phantoms are generated. Without loss of generality, the two fibers are assumed to reside in the $(x, y)$-plane. The equation that models the MRsignal then becomes:

$$
S(\tilde{\mathbf{g}})=S_{0}\left(f \exp \left(-b \tilde{\mathbf{g}}^{T} \mathbf{D}^{\mathbf{1}} \tilde{\mathbf{g}}\right)+(1-f) \exp \left(-b \tilde{\mathbf{g}}^{T} \mathbf{D}^{\mathbf{2}} \tilde{\mathbf{g}}\right)\right),
$$

with

$$
\mathbf{D}^{1,2}=\left(\begin{array}{ccc}
D_{x x}^{1,2} & D_{x y}^{1,2} & 0 \\
D_{x y}^{1,2} & D_{y y}^{1,2} & 0 \\
0 & 0 & D_{z}
\end{array}\right)
$$

where $f$ is the volume fraction of the first tensor $D_{z}$ is the same for both tensors. The gradient directions $\mathbf{g}$ are rotated to $\tilde{\mathbf{g}}$, such that the fiber bundles are 


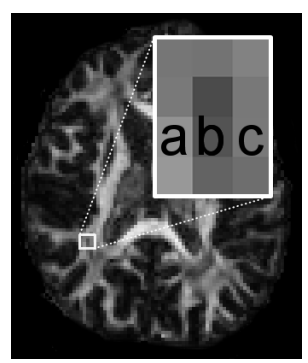

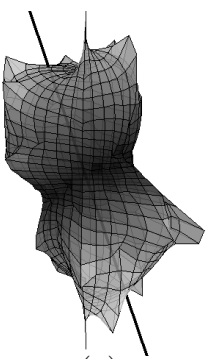

(a)

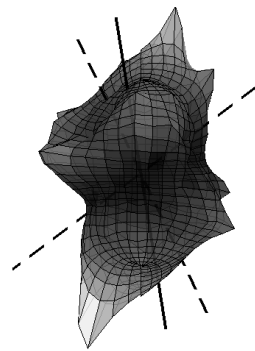

(b)

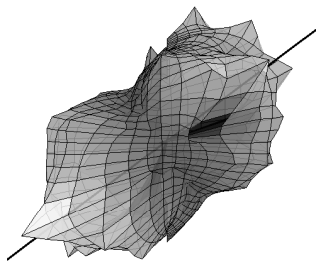

(c)

Fig. 1. Axial slice of the FA with logarithmic signal profiles of three adjacent voxels (a-c), measured at $b=3000 \mathrm{~mm} / \mathrm{s}^{2}$. Solid lines represent single tensor fits, dashed lines dual tensor fits to the data. Note the correspondence between the dashed lines in (b) and the solid lines in (a) and (c).

situated in the $(x, y)$-plane. The tensor components $D_{x z}^{1,2}$ and $D_{y z}^{1,2}$ are assumed to be negligible and are thus set to zero. Moreover, the diffusion in the direction perpendicular to the plane of interest is assumed to be equal for both fibers, i.e. the smallest eigenvalues of both tensors are equal. The data is therefore projected onto this direction. As a reference, the normal to the plane spanned by the principal diffusion directions in the voxels, used to generate the crossing, is used.

In measuring the diffusion profile of a crossing fiber, proper contrast is needed between the signal contributions of both fibers. Theoretically, altering the diffusion weighting parameter $b$ highly influences this contrast. In figure2a, a logarithmic signal profile $\left(\ln S_{0}-\ln S(\mathbf{g})\right)$ of a crossing fiber is computed for different $b$-values. The iso-lines further away from the origin (corresponding to high $b$ ) reveal more information about the crossing than the iso-lines near the origin. However, in measured data noise increasingly distorts measurements at higher $b$-values.

The angular resolution for different $b$-values will be determined in generated experimental phantoms, using the dual tensor method. The minimal angle between fiber bundles that can be estimated is defined at the point where both the bias and variance clearly start increasing.

\section{Results}

DTI-data of four healthy volunteers, between 20 and 30 years of age, were acquired on a Philips Intera 3.0 Tesla MRI scanner (Philips Intera, Philips Medical Systems, Best, The Netherlands) by means of a spin-echo EPI sequence. The diffusion weighting was along 92 three-fold tessellated icosahedric gradient directions. Other parameters were: TE $84 \mathrm{msec}$, TR $7912 \mathrm{msec}, b=1000,3000$ and $5000 \mathrm{~s} / \mathrm{mm}^{2}$, FOV $220 \mathrm{~mm}$, scan matrix 112 x 110, image matrix 128 x 128, slice thickness $3 \mathrm{~mm}$. Eddy current induced distortion was visually inspected to be negligible. 


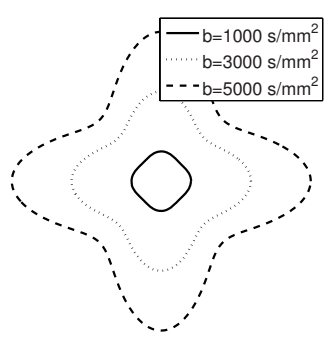

(a)

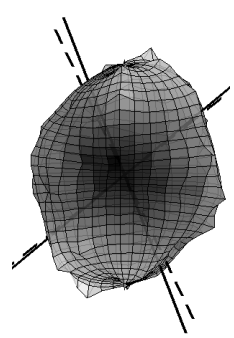

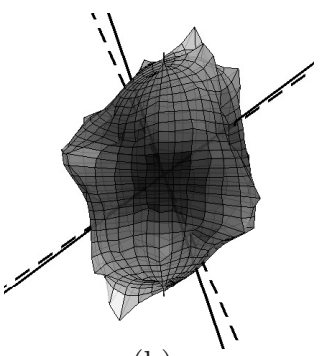

(b)

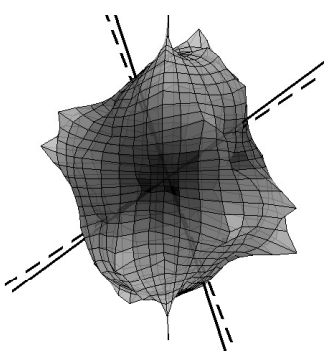

Fig. 2. (a) Computed logarithmic signal profiles for two perpendicular tensors with typical white matter properties [16] and (b) measured profiles after mixing voxels a and $\mathrm{c}$ in figure 1, for $b=1000,3000$ and $5000 \mathrm{~s} / \mathrm{mm}^{2}$ respectively. Solid lines represent single tensor fits before mixing, dashed lines dual tensor fits to the data after mixing. The profiles are scaled for proper visualization.

Only voxels for which FA > 0.6 were included. 1000 pairs of voxels were chosen to generate a uniform distribution of angles between the bundles in these voxels in the range $[0, \pi / 2]$. Next, the measured signals $S$ of these pairs were mixed, forming the crossing fiber phantoms.

For illustration purposes, logarithmic signal profiles of three adjacent voxels measured at $b=3000 \mathrm{~s} / \mathrm{mm}^{2}$ are displayed in figure 1. Here, the superior longitudinal fasciculus (a) and posterior corona radiata (c) are crossing (b). Logarithmic signal profiles after mixing DWIs of voxels a and $\mathrm{c}$ in figure 1 for $b=1000,3000$ and $5000 \mathrm{~s} / \mathrm{mm}^{2}$ are given in figure $2 \mathrm{~b}$.

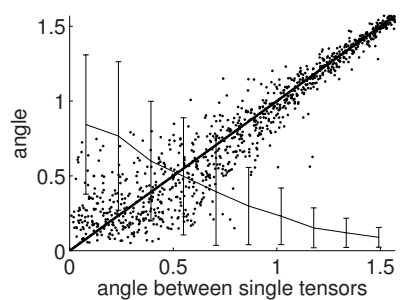

(a) $b=1000 \mathrm{~s} / \mathrm{mm}^{2}$

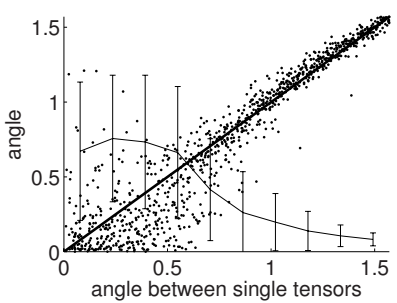

(b) $b=3000 \mathrm{~s} / \mathrm{mm}^{2}$

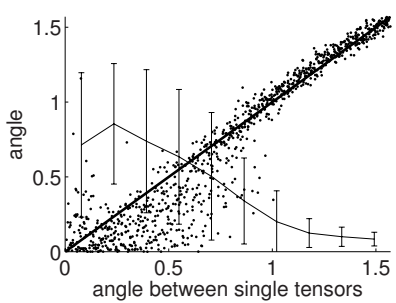

(c) $b=5000 \mathrm{~s} / \mathrm{mm}^{2}$

Fig. 3. Scatter plot of the estimated angle between the principal directions in the generated crossing fiber, as function of the angle between the single tensors. The diagonal solid bold line refers to ground truth. The solid line with errorbars denotes the error and standard deviation in the projection direction. Crossings were generated by mixing DWIs of two voxels with FA $>0.6$, for $b=1000,3000$ and $5000 \mathrm{~s} / \mathrm{mm}^{2}$ (a,b,c).

Optimization was performed based on the interior-reflective Newton method (Matlab, Mathworks) [17. A quadratic regularization term for the volume fraction $f$ was added to the cost function, with a scaling factor of 0.2 around the mean value 0.5 . The results of fitting the generated crossing fibers for $b=1000$, 


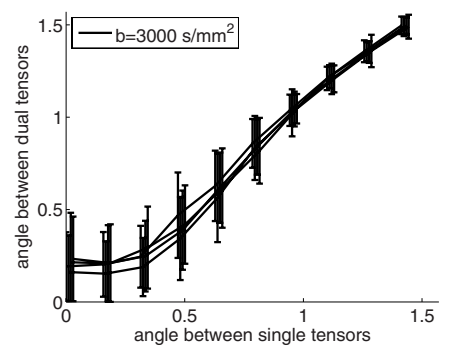

Fig. 4. Estimated mean angle with errorbars denoting the standard deviation, as function of the input angle for $b=3000 \mathrm{~s} / \mathrm{mm}^{2}$ for four datasets of healthy individuals.

3000 and $5000 \mathrm{~s} / \mathrm{mm}^{2}$ are displayed in figure 3 . The reproducibility of the method was analyzed by computing the angular deviation with respect to the input angle for data with $b=3000 \mathrm{~s} / \mathrm{mm}^{2}$ of four healthy individuals, depicted in figure 4.

An angular resolution of $0.6 \pi$ was found, for both $b=1000,3000$ and 5000 $\mathrm{s} / \mathrm{mm}^{2}$. Reproducible results in four subjects were achieved.

Finally, a single streamline was generated in the body of the corpus callosum, passing through the crossing with the corticospinal tract [3. This streamline incorrectly follows the indermediate orientation of the two fiber bundles. A dual tensor fit was performed along this tract, and estimated fiber bundle orientations were plotted. The decreased FA and increased planar tensor component $c_{p}$ of the single tensor indicate the presence of a crossing. This is confirmed by the higher FAs of the dual tensors along the tract. The results of this experiment are given in figure 5 .

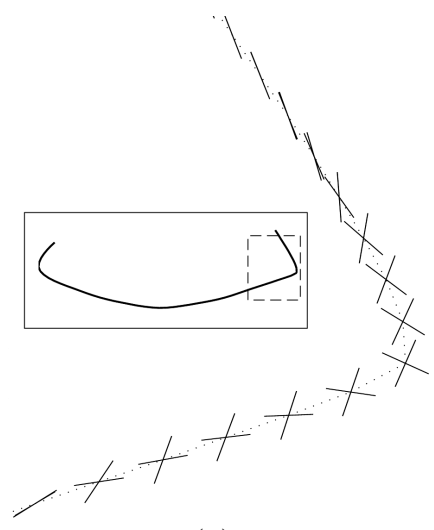

(a)

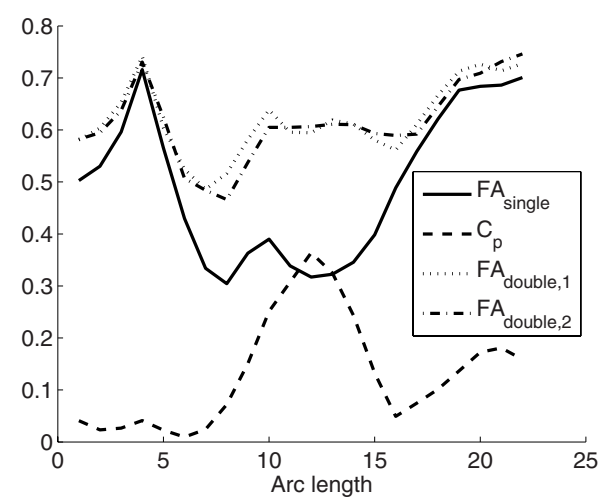

(b)

Fig. 5. (a) Selected part of a single streamline in the body of the corpus callosum (inset), with solid lines representing the principal directions after a dual tensor fit, and (b) anisotropy measures based on single $\left(\mathrm{FA}_{\text {single }}, c_{p}\right)$ and dual tensor fit $\left(\mathrm{FA}_{\mathrm{dual}, 1}\right.$, $\mathrm{FA}_{\text {dual,2 }}$ ) along this tract 


\section{Discussion}

We propose a new method for creating experimental phantom data of fiber crossings, by mixing the DWI-signals from high FA-regions with different orientation. The properties of these experimental phantoms approach the conditions of real data. These phantoms can thus serve as a 'ground truth' in validating crossing reconstruction algorithms. In this paper, the angular resolution of a dual tensor model is determined. Series of crossings, generated under different angles, give insight into the precision that can be achieved in estimating the fiber bundle orientations. Not only are the results based on realistic imaging artifacts, but also can the reconstructed fiber orientations be compared to a ground truth, being the single tensor fits on the individual voxels.

An equal angular resolution of $0.6 \pi$ was found, for both $b=1000,3000$ and $5000 \mathrm{~s} / \mathrm{mm}^{2}$. A higher resolution for higher $b$-values was to be expected, due to more non-linear behaviour of the diffusion profile. Apparently, the decreased SNR in high $b$-value data (with constant scanning time), due to a stronger signal attenuation, strongly affected the algorithm's performance. Using a Rician noise model in a Maximum Likelihood estimator might reduce the bias in the reconstructed angle. The error in the estimated projection direction clearly increases for lower input angles, resulting in an ill-defined crossing fiber plane. Nevertheless, the deviation in estimated output angles did not decrease significantly when the correct projection direction was used instead.

Future work consists of acquiring DTI-data at $b$-values around $1000 \mathrm{~s} / \mathrm{mm}^{2}$, to determine the optimal $b$-value in maximizing the angular resolution in the reconstruction of crossing fibers. Building a theoretical model will aid in choosing proper values for $b$ and other imaging parameters, the number of repetitive measurements and gradient directions. Eventually, tractography with increased precision and an unbiased anisotropy estimation will lead to an improved analysis of both healthy and pathological brain data.

Instead of generating phantoms out of magnitude data, the real and imaginary part of the DWIs could be individually mixed. A Gaussian noise model could then be obtained in both parts, making even more realistic experimental phantoms. However, phase distortions, between and within acquisitions, hamper usage of the complex data. Until now, no correction method for these distortions is available.

The proposed validation method is expected to be useful in various crossing reconstruction algorithms. As a result, crossing fiber regions can be reliably included in clinical studies to brain diseases.

\section{Acknowledgments}

The involvement of M.W.A. Caan took place in the context of the Virtual Laboratory for e-Science project (http://www.vl-e.nl/), is supported by a BSIK grant from the Dutch Ministry of Education, Culture and Science (OC\&W) and is part of the ICT innovation program of the Ministry of Economic Affairs (EZ). 


\section{References}

1. Basser, P., Mattiello, J., Bihan, D.L.: Estimation of the effective self-diffusion tensor from the NMR spin echo. J. Magn. Reson. B(103), 247-254 (1994)

2. Basser, P.: Inferring microstructural features and the physiological state of tissues from diffusion-weighted images. NMR Biomed. 8, 333-334 (1995)

3. Blaas, J., Botha, C., Peters, B., Vos, F., Post, F.: Fast and reproducible fiber bundle selection in dti visualization. In: Proc. IEEE Visualization, pp. 59-64 (2005)

4. Corouge, I., Fletcher, P.T., et al.: Fiber tract-oriented statistics for quantitative diffusion tensor MRI analysis. Med. Im. Anal. 10, 786-798 (2006)

5. Tuch, D.: Diffusion MRI of complex tissue structure. PhD thesis (2002)

6. Frank, L.: Anisotropy in high angular resolution diffusion-weighted MRI. Magn. Reson. Med. 45, 935-939 (2001)

7. Kreher, B., Schneider, J., et al.: Multitensor approach for analysis and tracking of complex fiber configurations. Magn. Res. Med. 54, 1216-1225 (2005)

8. Frank, L.R.: Characterization of anisotropy in high angular resolution diffusionweighted MRI. Magn. Reson. Med. 47, 1083-1099 (2002)

9. Özarslan, E., Mareci, T.H.: Generalized diffusion tensor imaging and analytical relationships between diffusion tensor imaging and high angular resolution diffusion imaging. Magn. Reson. Med. 50, 955-965 (2003)

10. Peled, S., Friman, O., et al.: Geometrically constrained two-tensor model for crossing tracts in DWI. Magn. Reson. Med. 24, 1263-1270 (2006)

11. Alexander, D., Barker, G., et al.: Optimal image parameters for fiber-orientation estimation in diffusion MRI. NeuroImage 27, 357-367 (2005)

12. Assaf, Y., Freidlin, R., et al.: New modeling and experimental framework to characterize hindered and restricted water diffusion in brain white matter. Magn. Reson. Med. 52, 965-978 (2004)

13. Anderson, A.W.: Theoretical analysis of the effects of noise on diffusion tensor imaging. Magn. Reson. Med. 46, 1174-1188 (2001)

14. Gudbjartsson, H., Patz, S.: The Rician distribution of noise MRI data. Magn. Res. Red. 910-914 (1995)

15. Peled, S., Westin, C.F.: Geometric extraction of two crossing tracts in DWI. Proc. Intl. Soc. Mag. Reson. Med. 13, 1 (2005)

16. Pierpaoli, C., Jezzard, P., et al.: Diffusion tensor MR imaging of the human brain. Radiology 201, 637-648 (1996)

17. Coleman, T.F., Li, Y.: An interior trust region approach for nonlinear minimization subject to bounds. SIAM J. Optim. 6, 418-445 (1996) 